\title{
EDITORIAL
}

\section{Expert consensus on COVID-19 vaccination in children}

\author{
Yue-Jie Zheng ${ }^{1} \cdot$ Xiao-Chuan Wang $^{2} \cdot$ Lu-Zhao Feng $^{3} \cdot$ Zheng-De Xie $^{4} \cdot$ Yi Jiang ${ }^{5} \cdot$ Gen Lu $^{6} \cdot$ Xing-Wang Li $^{7}$.

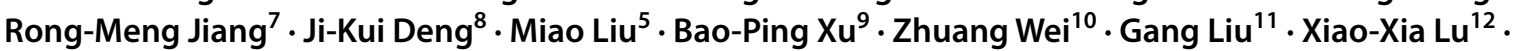 \\ Run-Ming Jin ${ }^{13}$. Zhi-Sheng Liu ${ }^{14}$. Yun-Xiao Shang ${ }^{15}$. Sai-Nan Shu ${ }^{16}$. Yan Bai ${ }^{13}$. Min Lu ${ }^{17}$. Guang-Hua Liu ${ }^{18}$. \\ Wan-Jun Luo ${ }^{19} \cdot$ Yu-Xia Cui ${ }^{20} \cdot$ Le-Ping Ye ${ }^{21} \cdot$ Li-Kai Lin $^{22}$ • Dong-Chi Zhao ${ }^{23}$. A-Dong Shen ${ }^{24}$. Jian-Bo Shao ${ }^{25}$. \\ Li-Juan Xiong $^{26}$ • Li-Wei Gao ${ }^{9}$ Tian-You Wang ${ }^{27}$. China National Clinical Research Center for Respiratory Diseases . \\ National Center for Children's Health, Beijing, China - Group of Respirology, Chinese Pediatric Society, Chinese \\ Medical Association - Chinese Medical Doctor Association Committee on Respirology Pediatrics - China Medicine \\ Education Association Committee on Pediatrics - Chinese Research Hospital Association Committee on Pediatrics . \\ Chinese Non-government Medical Institutions Association Committee on Pediatrics - China Association of \\ Traditional Chinese Medicine, Committee on Children's Health and Medicine Research · Zheng-Yan Zhao ${ }^{28}$. \\ Yong-Hong Yang ${ }^{24} \cdot$ Kun-Ling Shen ${ }^{9}$
}

Received: 31 August 2021 / Accepted: 13 September 2021 / Published online: 7 October 2021

(c) Children's Hospital, Zhejiang University School of Medicine 2021

Coronavirus disease 2019 (COVID-19) is a public health disaster that has not been encountered for a hundred years. On January 12, 2020, the World Health Organization (WHO) confirmed and named the coronavirus which caused unexplained pneumonia as 2019 novel coronavirus (2019$\mathrm{nCoV})$. Since then, the disease caused by this virus had been named as coronavirus disease 2019 (COVID-19). On February 11, 2020, the International Committee on Taxonomy of Viruses announced that the English name of the novel coronavirus was severe acute respiratory syndrome coronavirus 2 (SARS-CoV-2) [1]. At present, SARS-CoV-2 infection is still rampant worldwide. As of September 10, 2021, there were about 222 million confirmed cases of COVID-19 and more than 4.5 million deaths worldwide [2]. According to the American Centers for Disease Control and Prevention, by July 29, 2021, 4.19 million pediatric COVID-19 cases had been reported in the United States, accounting for $14.3 \%$ of all confirmed cases in the United States, with a cumulative reported incidence of 5.6\% [3]. Among children infected with COVID-19, $0.1 \%-1.9 \%$ cases require hospitalization, with a mortality rate of $0.00-0.03 \%$ [3]. WHO surveillance data showed that the overall mortality rate of confirmed cases with COVID-19 in the whole population ranges from $1 \%-3 \%$, and the mortality rate under 5 years of age is approximately 7 per million [4]. However, the Delta variant has caused a surge in COVID-19 cases in children. Once infected, the incidence of severe disease is $1 \%$ and the

Zheng-Yan Zhao

zhaozy@zju.edu.cn

Extended author information available on the last page of the article rate of death is $1 / 10,000$ [5], which posing serious threat to the lives and health of children. With the development of COVID-19 vaccines and the gradual vaccination worldwide, the increasing number of cases in unvaccinated children and adolescents has received attention. According to WHO surveillance data, the proportion of COVID-19 cases in children gradually increased, with the proportion of cases in the age groups of less than 5 years and 5-14 years increasing from $1.0 \%$ and $2.5 \%$ in January 2020 to $2.0 \%$ and $8.7 \%$ in July 2021, respectively [4].

At the early stage of the COVID-19 epidemic, most of the pediatric cases with COVID-19 were family clustered, that is, the source of infection in children was mostly the adult patients in the family. With the widespread COVID-19 vaccination in adults and the increase in pediatric infections, pediatric infections may become an important source of community transmission of COVID-19. In addition, children are also an important part of the whole population to build the immune barrier of COVID-19; as minors, children infected with COVID-19 bring a heavy burden on families and society; COVID-19 infection can also cause severe and fatal cases in children. Therefore, the progressive vaccination in the pediatric population against the COVID-19 is important for the overall prevention and control of COVID19. Preliminary studies have shown that the COVID-19 vaccine is safe and effective after vaccination in children, and the COVID-19 vaccination has been started in children over 12 years of age in the United States since May 2021. China has approved the emergency use of inactivated SARSCoV-2 vaccine for children aged 3-17 years since July 2021 [6]. Experts were organized to study and formulate specific 
vaccination policies for children aged 3-17 years according to the needs of epidemic prevention and control. Vaccination in children across the country is also organized and implemented by provinces and municipalities according to the principle of advancing in a gradual manner, highlighting priorities, ensuring safety, and advancing the population in order.

For implementation of COVID-19 vaccination in children stably, academic institutions, including National Clinical Research Center for Respiratory Diseases, National Center for Children's Health, and Chinese Pediatric Society of Chinese Medical Association organize relevant experts to reach this consensus on COVID-19 vaccination in children.

\section{Safety, efficacy and accessibility of COVID-19 vaccines}

SARS-CoV-2 is a spherical single-positive-sense ribonucleic acid (RNA) virus, and the main structures of this virus include single-stranded positive-sense RNA (ssRNA), spike protein (S), membrane protein (M), envelope protein (E), and nucleocapsid protein $(\mathrm{N})$. Studies have shown that $\mathrm{S}$ protein is responsible for recognizing and binding the receptor on the surface of host cells and plays an important role in the invasion process of viral infection. It is composed of $\mathrm{S} 1$ and $\mathrm{S} 2$ subunits. The receptor-binding domain (RBD) of the $\mathrm{S} 1$ subunit can recognize and bind the host cell surface receptor angiotensin-converting enzyme 2 (ACE2). The conformational change of the $\mathrm{S} 2$ subunit mediates the fusion between the viral envelope and the host cell membrane, causing viral infection of host cells. Therefore, $\mathrm{S}$ protein is an important target for vaccine research and development [7]. After vaccination, the body will produce antiviral antibodies, of which the antiviral activity of neutralizing antibody mainly blocks the binding of SARS-CoV-2 to ACE2, by binding to the receptor-binding domain RBD of the $S$ protein. Several COVID-19 vaccines are currently marketed $[8,9]$ : inactivated vaccines, viral vector vaccines, recombinant protein vaccines, and nucleic acid vaccines. The characteristics and mechanisms of various vaccines are as follows: (1) inactivated SARS-CoV-2 vaccines: The virus is cultured in vitro, and inactivated by heating or chemical methods, to eliminate its pathogenicity and retains antigenicity. In China, the inactivated SARS-CoV-2 vaccine is produced by culturing SARS-CoV-2 in African green monkey kidney (Vero) cells, inactivation and addition of aluminum hydroxide adjuvant to enhance immunogenicity and stimulate the immune response; (2) viral vector vaccines: the vaccine is prepared by inserting the $S$ protein gene into the modified non-pathogenic live viral vector. The adenovirus vector COVID-19 vaccine (adenovirus vector type 5) in China is produced by recombining the $\mathrm{S}$ protein gene into the replication-deficient human adenovirus type 5 genome; and the recombinant adenovirus expresses the $\mathrm{S}$ protein antigen in vivo to induce the immune response in the body; (3) recombinant protein vaccines: specific parts or subunits with immunogenicity in pathogens are used to prepare the vaccine by genetic engineering. The recombinant SARS-CoV-2 vaccine ( $\mathrm{CHO}$ cell) in China is produced by recombining the DNA sequence encoding the RBD domain of SARSCoV-2 directly involved in host receptor recognition into the genome of Chinese hamster ovary (CHO) cells. The RBD dimer is expressed in vitro, and then aluminum hydroxide adjuvant is used to improve the immunogenicity and induce the immune response; (4) nucleic acid vaccines: mRNA vaccines and DNA vaccines delivering the genetic material encoding $\mathrm{S}$ protein into the cells near the injection site, and host cells were utilized to synthesize $S$ protein to activate the immune response. At present, mRNA vaccines are mainly used in clinical applications.

Up to now, there are seven COVID-19 vaccines which have been approved for conditional marketing or emergency use in China, including three inactivated vaccines and one adenovirus vector vaccine (adenovirus vector type 5) for conditional marketing, and one recombinant COVID-19 vaccine ( $\mathrm{CHO}$ cells) and two inactivated vaccines for emergency use. The COVID-19 vaccines approved by WHO for emergency use include the inactivated vaccine (BBIBPCorV) of China Sinopharm CNBG Beijing Institute of Biological Products (hereinafter referred to as "CNBG") and the inactivated vaccine (CoronaVac) of Sinovac Biotech Co., Ltd. (hereinafter referred to as "Sinovac").

The inactivated vaccine of CNBG (BBIBP-CorV) and the inactivated vaccine of Sinovac (CoronaVac) have good immunogenicity, protective efficacy and safety in adults. A Phase III clinical trial of BBIBP-CorV vaccine [10] showed that the positive conversion rate of neutralizing antibodies in the subject group after two doses of vaccine was $100 \%$, and the vaccine protective efficacy was $78.1 \%$. The safety was good, and adverse reactions were mild. Pain at the injection site was the most common adverse reaction. A Phase I/II clinical trial on CoronaVac vaccine showed [11] that the positive conversion rate of neutralizing antibodies exceeded $90 \%$ after 28 days of full vaccination, with good safety and mild adverse effects. The results of another real-world study [12] showed that after full vaccination with CoronaVac, the protection rate was $65.9 \%$ for symptomatic COVID-19 and 87.5\% for prevention of hospitalization.

At present, billions of adults worldwide have been vaccinated with various COVID-19 vaccines. The vaccines have been proved to be safe and protective in reducing infection and transmission rate, risk of severe disease, hospitalization and death. A double-blind randomized controlled Phase I/ II clinical trial on the safety, tolerability and immunogenicity of inactivated SARS-CoV-2 vaccine (CoronaVac) in 550 
children and adolescents aged 3-17 years (including 143 children aged 3-5 years, 204 children aged 6-11 years and 203 children aged 12-17 years) have been carried out in China [13]. The results showed that the positive conversion rate of neutralizing antibodies in vaccinated children reached $100 \% 28$ days after full vaccination with two doses of the vaccines. The geometric mean titer (GMT) of antibody reached at 117.4 while no neutralizing antibodies were detected in the placebo group; there was no significant difference in immune response among different age groups of 3-5 years, 6-11 years and 12-17 years, all of which were superior to adults aged 18-59 years and the elderly aged 60 years and above. Adverse reactions to vaccination were $29 \%(63 / 217)$ and $24 \%$ (27/114) in the vaccine group and the control group, respectively. The adverse reactions were mainly mild and moderate and no serious adverse reactions were observed. Injection, site pain (13\%) was the most common manifestation. Another international multicenter, placebo-controlled, observer-blind Phase III clinical trial of 2260 people aged $12-15$ years old with COVID-19 mRNA vaccine (BNT162b2) [14] also confirmed that higher neutralizing antibody response after vaccination in children; and the adverse reactions of vaccination were mainly transiently mild to moderate reactions, including injection site pain (79-86\%), fatigue (60-66\%) and headache (55-65\%), without serious adverse events related to vaccination. Clinical trials of COVID-19 vaccines in children aged 6 months to 17 years have been initiated and results will be reported soon.

At present, multiple mutations of SARS-CoV-2 have occurred around the world. Many countries have successively experienced epidemic peaks again. Recently, the phenomenon of vaccine breakthrough infection has been reported, that is, infections that occur 14 days after completion of the full course of vaccination. A real-world study of 1497 medical staff in Israel who were completely vaccinated with BNT162b2 (Pfizer/BioNTech) vaccine showed [15] that after the full course of vaccination with BNT162b2, only 39 medical staff had breakthrough infection, all of which were mild or asymptomatic, and the GMT of perioperative neutralizing antibodies in patients with breakthrough infection was significantly lower than that in the control group. Considering that the local Alpha variant strain was predominantly prevalent and there was a local outbreak of Delta variant strain in the late stage of the trial, it was suggested that the vaccine might have some protective effect against the variant strain. The results of a study conducted in the outbreak of the Delta variant of the COVID-19 in Guangzhou, China, in May 2021 [16] showed that the domestic inactivated vaccine was protective against the Delta variant with an overall protection rate of 59\%, 70.2\% against moderate symptoms, and $100 \%$ against severe disease. The above results suggest that despite the continuous emergence of COVID-19 variants, the existing COVID-19 vaccines still have a good protective ability against variants.

\section{Application of COVID-19 vaccine in children}

Achieving herd immunity against COVID-19 through vaccination rather than through natural infection and transmission of the disease in the population is the best strategy to terminate the current pandemic of COVID-19. Herd immunity against COVID-19 is not achievable without the widespread vaccination of children and adolescents, because they account for more than one fourth of the total population. According to the statistics, children account for $14.3 \%$ of those diagnosed with COVID-19 in the United States. Although the clinical manifestations of children with COVID-19 are usually mild or asymptomatic compared with adults, a small proportion of patients are severe, which leading to hospitalization and even death. Recent surveillance in the United States has shown an increase in morbidity and severe cases in children, which must be of great concern. In addition, acute respiratory infections are the most common illnesses in children, and the manifestations of COVID-19 in children are difficult to be distinguished from other respiratory infections. Infected children may play a more important role in community transmission (including families, childcare facilities, and schools) as a source of infection. Children are, therefore, an important population who need COVID-19 vaccination. In view of the safety, efficacy and accessibility of the COVID-19 vaccines, as well as the impact of the COVID-19 on the health of people of all ages, the population of the COVID-19 vaccination are started from adults, and then promoted to adolescents.

Seven COVID-19 vaccines have been approved by WHO for emergency use in children (Table 1) [24]. China has approved the emergency use of inactivated COVID-19 vaccines in children aged 3-17 years [25]. Canada, the United States, Europe, Singapore, the United Arab Emirates, Kuwait and other countries have also approved emergency vaccination with COVID-19 vaccine in children and adolescents aged 12-17 years [26]. As of August 8, 2021, nearly 10 million children aged 12-17 years in the United States have completed COVID-19 mRNA vaccination. More than 60 million doses of vaccination have been administered to children aged 12-17 years in China [27].

The immune system of children, especially infants, is in the process of continuous development and improvement, and the relevant application data of COVID-19 vaccines are limited at present, which needs to be confirmed by more clinical trials. It is recommended that COVID-19 vaccination in children be advanced gradually from older to younger ages under an orderly manner, and the effectiveness of vaccination and adverse reactions should be closely monitored. 
Table 1 Seven COVID-19 vaccines approved by WHO for emergency use [17-24]

\begin{tabular}{llllll}
\hline Vaccine name & Date of approval & Manufacturer & Country of origin & Vaccine type & Protective efficacy \\
\hline BNT162b2 & January 14, 2021 & Pfizer/BioNTech & United States/Germany & mRNA vaccine & $95 \%$ \\
mRNA-1273 & February 03, 2021 & Moderna & United States & mRNA vaccine & $94.1 \%$ \\
AZ1222 & March 01, 2021 & AstraZeneca/Univer- & United Kingdom & Adenoviral vector vaccine & $63.09 \%$ \\
& sity of Oxford & & & \\
Covishield & March 01, 2021 & Serum institute of & India & Adenoviral vector vaccine & $63.09 \%$ \\
& & India & & & \\
Ad26.COV2.S & March 17, 2021 & Johnson \& Johnson & United States & Adenoviral vector vaccine & $66.9 \%$ \\
BBIBP-CorV & May 07, 2021 & CNBG & China & Inactivated vaccine & $78.1 \%$ \\
CoronaVac & June 01, 2021 & Sinovac & China & Inactivated vaccine & $50.7 \%$ \\
\hline
\end{tabular}

\section{Procedures and general principles for COVID-19 vaccination in children}

The current vaccine approved for emergency use in children in China is inactivated SARS-CoV-2 vaccine, which is administered in two doses. The vaccination interval between two doses is recommended to be $\geq 3$ weeks. The second dose should be completed as early as possible within 8 weeks. The dose and method used are the same as those for adults. The vaccines approved for use in children abroad are mRNA vaccines with two doses and a recommended interval of $\geq 3$ weeks between the two doses. Recent studies have shown that booster vaccination with the COVID-19 vaccine provides longer term protection, but booster vaccination is not recommended for children at this time.

Principle of delayed vaccination and catch-up vaccination of COVID-19 vaccines [28]: for those who have not completed the full-course COVID-19 vaccination, it is recommended that the full course of vaccination be completed as soon as possible by giving a catch-up vaccination. The immunization program does not need to be restarted during revaccination, and the corresponding dose can be completed. It is recommended to complete catch-up vaccination with the same vaccine product. In the event that the COVID-19 vaccines cannot be supplied continually or the vaccine recipient is vaccinated in another place and other special circumstances make it impossible to complete the vaccination with the same vaccine product, the same type of vaccine product from other manufacturers can be used to complete catch-up vaccination. Although recent studies have confirmed that mixed administration of adenoviral vector vaccine (ChAdOx $1 \mathrm{nCoV}-19)$ with mRNA vaccine (BNT162b2 or mRNA-1273) can achieve better immunoreactivity in adults $[29,30]$, mixed administration or catch-up vaccination with different kinds of COVID-19 vaccines is not recommended for the time being.

\section{The principle of simultaneous vaccination with planned immunization vaccines and other self-funded vaccines}

In China, simultaneous vaccination with other vaccines is not recommended for the time being, and the vaccination interval between other vaccines and COVID-19 vaccines should be longer than 14 days [28]. When rabies vaccine, tetanus vaccine, rabies or tetanus immunoglobulin are required to be vaccinated due to animal injury, trauma or other reasons, the vaccination interval with the COVID-19 vaccine could not be considered.

\section{Indications and contraindications for COVID-19 vaccination in children}

COVID-19 vaccination for children of appropriate age needs to be implemented according to the vaccine instructions. If it is not mentioned in the package insert of this vaccine, it needs to be implemented according to the vaccination guidelines issued by the national competent authority. If not mentioned in the above guidance document, the responsible body for the specific administration may refer to the professional literature as appropriate.

\section{Appropriate population for COVID-19 vaccination}

1. All healthy children of appropriate age (12-17 years old). With the gradual improvement of evidence-based medical evidence on safety and effectiveness, the vaccination plan for young children aged 3-12 years old is further determined and promoted orderly according to the relevant national vaccination guidelines of COVID-19 vaccination.

2. Children of appropriate age in the stable phase of chronic disease (at least 3 months, including use of nonimmunosuppressive drugs). 
3. For those who use medium and low-dose glucocorticoids and most biological agents (except rituximab and other preparations against B cells) and have stable disease for at least 3 months, vaccination should be implemented during the interval between drug use as far as possible.

\section{Population for delayed vaccination}

1. Children who are under acute phase of various diseases or active phase of chronic diseases. After recovering from acute diseases and chronic diseases stabilizing for 3 months, vaccination may be considered.

2. Children during the period of use of some immunosuppressive drugs (including immune-related biologics).

(1) One month after the end of high-dose glucocorticoid (prednisone $\geq 20 \mathrm{mg} /$ day or $>2 \mathrm{mg} / \mathrm{kg} /$ day) treatment.

(2) For patients treated with rituximab: 5 months after the last dose or before $\mathrm{B}$ cells counting returns to normal.

3. Patients receiving chemotherapy for leukemia: within 6 months of the end of chemotherapy.

4. Patients undergoing hematopoietic stem cell transplantation. Vaccination should be suspended within 1 year after transplantation, and vaccination should be performed only after the immune function is normal 1 year later.

\section{Contraindicated population for vaccination}

1. Children with allergic reactions to the relevant components contained in the COVID-19 vaccine.

2. Children with previous severe allergic reactions to vaccines (such as acute allergic reactions, angioedema, dyspnea, etc.).

3. Children who have had adverse reactions to COVID-19 vaccination (excluding low-grade fever, local swelling and pain and other general reactions).

\section{Recommendations for COVID-19 vaccination in children with other special health conditions}

\section{Children suffering from the following allergic diseases can be vaccinated in stable status}

Children who are allergic to dust mites, pollen, food (eggs, peanuts, seafood, mango, etc.), alcohol, penicillin, cefotaxime, and who suffer from allergic rhinitis, allergic conjunctivitis, atopic dermatitis and food allergy.

\section{Children with asthma}

Bronchial asthma is not a contraindication to COVID-19 vaccination. Vaccination should be carried out in the remission stage of asthma (including inhaled corticosteroids). In acute asthma attacks (wheezing, cough, shortness of breath, chest tightness and other symptoms), especially when glucocorticoid is used systemically (including oral and intravenous administration), vaccination should be suspended. COVID-19 vaccine may be administered during anti-IgE monoclonal antibody therapy and allergen-specific immunotherapy, but they should not be performed on the same day [31].

\section{Children with impaired immune function}

In principle, children with congenital or acquired impaired immune function can be vaccinated with inactivated SARS$\mathrm{CoV}-2$ vaccine, which usually has the same safety as immunocompetent children; however, the intensity and persistence of immune protection will be reduced after vaccination in children with impaired immune function.

\section{Children who have been previously diagnosed with COVID-19}

In principle, one dose of the COVID-19 vaccine can be administered after 6 months of infection [28].

\section{Use of intravenous immunoglobulins}

The use of intravenous immunoglobulins may affect the efficacy of some live attenuated vaccines, but has no effect on inactivated SARS-CoV-2 vaccines [32].

\section{Adverse reactions and treatment after COVID-19 vaccination in children $[8,13$, 14, 33-36]}

\section{Common adverse reactions and treatment}

Most of the adverse reactions of the COVID-19 vaccination are mild to moderate and resolved within $24 \mathrm{~h}$ after vaccination. Common adverse reactions include pain, redness and swelling at the injection site as well as fever, headache, nausea, diarrhea, fatigue and limb pain $[8,13,14]$. The incidence of adverse reactions varies after vaccinations with different COVID-19 vaccines. For example, the incidence of adverse reactions in Phase I/II clinical trials of inactivated SARS-CoV-2 vaccine 
was $15.0 \%$, and the most common adverse reactions were local pain and fever. The incidence of adverse reactions in Phase II/ III clinical trials of SARS-CoV-2 mRNA vaccine (BNT162b2, Pfizer/BioNTech) was $27.0 \%$, and the most common adverse reactions were local pain and fatigue [33]. The incidence of adverse reactions in the Phase I clinical study of SARS-CoV-2 recombinant spike protein vaccine (SCB-2019, Clover Biopharmaceutical) was $34.7 \%$, and the most common adverse reactions were local pain and headache [34]. According to the report of Chinese Center for Disease Control and Prevention [35], large-scale population vaccination has been initiated in China since December 15, 2020. As of April 30, 2021, 265 million doses of COVID-19 vaccine have been reported, and the reported incidence of adverse reactions is $11.86 / 100,000$ doses. Among them, general reactions accounted for $83 \%$, and the reported incidence was 9.84/100,000 doses; abnormal reactions accounted for $17 \%$, with a reported incidence of 2.02/100,000 doses; the incidence of serious abnormal reactions was $0.07 / 100,000$ doses, and the probability of occurrence was within the range of very rare (less than 1:10,000). The incidence of general reactions and abnormal reactions after vaccination with COVID-19 vaccines in China are lower than the average reporting level of other types of vaccines in China in 2019 [36].

The common adverse reactions after vaccination with COVID-19 vaccines are similar to those after other vaccinations. In most cases, these adverse reactions are mild and often related to the reactogenicity of the body to the vaccine, reflecting the normal innate immune response of the body to antigen exposure. Published data show that the COVID19 vaccine has transient local reactogenicity, such as pain, redness, swelling, and induration, and systemic reactogenicity, such as fever, chills, myalgia, and headache. The above adverse reactions can be relieved by paracetamol or other antipyretic and analgesic drugs.

\section{Possible special adverse reactions and treatment}

\section{Severe allergic reaction}

Severe allergic reaction most commonly occurs when vaccinated with the first dose of COVID-19 mRNA vaccine, mainly manifesting as nausea, vomiting, facial flushing, rash, laryngeal edema, wheezing, tachycardia, and even hypotension, heart failure, and anaphylactic shock. In terms of the SARS-CoV-2 mRNA vaccine, the greatest potential high risk component for inducing allergic reactions is polyethylene glycol (PEG). The mechanism of PEG-induced allergic reactions is not yet fully understood and may involve IgEand non-IgE-mediated anaphylaxis. The adenoviral vector SARS-CoV-2 vaccines recently authorized for emergency use do not contain PEG, but contain structurally related polysorbate- 80 and also require vigilance for cross-reactivity. For children with allergic reactions with mild symptoms, appropriate observation can be given; for vaccinated children with severe allergic reactions, relevant allergic conditions should be treated according to the emergency treatment protocol in the Guidelines for Pediatric Allergy. First-line drugs such as epinephrine should be used in time, and circulation, airway, respiration, skin, etc. should be assessed by specialists, so that the emergency process can be quickly initiated.

\section{Other special adverse reactions}

Other specific adverse reactions have been reported mainly for SARS-CoV-2 mRNA vaccines. (1) Extensive limb swelling (ELS) and arthurs reaction: ELS is a common special adverse reaction of vaccination at all ages. Arthurs reaction occurs in a very small proportion of vaccines, manifesting as edema, hemorrhage, and necrotizing vasculitis, ulcers at the injection site and even anaphylactic shock; (2) myocarditis: to date, there have been relatively few reports on the development of myocarditis after COVID-19 vaccination, mainly in adolescents and young adult males; (3) thrombosis with thrombocytopenia syndrome (TTS): TTS is a very rare adverse reaction after vaccination, mainly occurs in adult women. It is manifested as severe or persistent headache, blurred vision, shortness of breath, chest pain, leg swelling or petechiae, etc. There is no clear causal relationship between TTS and COVID-19 vaccination; (4) Transverse myelitis and Guillain-Barré syndrome (GBS): there are suspected transverse myelitis manifestations such as headache, chest and back pain, generalized weakness and urinary retention after COVID-19 vaccination. In addition, GBS has also been reported after COVID-19 vaccination, manifested as weakness of both lower limbs and paresthesia. Neurological adverse effects after vaccination with COVID-19 vaccine are very rare, and a causal relationship with COVID-19 vaccination cannot be clarified. In conclusion, the above special adverse reactions are very rare, and it is not clear whether these will occur after vaccination in children. However, COVID-19 vaccination is the first priority of global public health cause. It is very important to monitor these potential serious adverse reactions; and we should also be highly vigilant during the vaccination in children. 


\section{Other issues of COVID-19 vaccination in children $[28,35,36]$}

\section{Precautions after COVID-19 vaccination in children}

1. After vaccination with COVID-19 vaccine, children should be observed at the vaccination site for at least $30 \mathrm{~min}$ to observe whether there is acute allergic reaction, syncope and other conditions. During the observation period, children are not allowed to gather or play.

2. After vaccination, keep the skin of vaccination site clean and dry, avoid scratching the vaccination site by hand, avoid excessive compression and stimulation when bathing, and keep injection site cleaning after bathing.

3. Normal activity and diet are allowed in children after vaccination with the COVID-19 vaccine, but strenuous exercise and exposure to known allergens should be avoided. Ensure good rest adequate sleep in children after vaccination.

4. Avoid implying and inducing adverse reactions in children, and closely observe their physical condition and body temperature changes. If fever persists or serious adverse reactions occur, report to the vaccination site and seek prompt medical attention.

5. The antibody test used clinically for SARS-CoV-2 is different from the neutralizing antibody test used for the virus neutralization test. It is not recommended to use the clinical antibody test to determine the effectiveness of vaccination. Antibodies produced after COVID-19 vaccination also do not affect clinical diagnosis.

\section{Routine protective measures after COVID-19 vaccination in children}

1. Avoiding infection in children after COVID-19 vaccination. Children should still take protective measures include wearing a mask, frequent hand washing, good personal hygiene, and maintaining social distance.

2. Children still should be wear masks and replaced in a timely manner in crowded places, public places and closed places or when in contact with patient after vaccination.,

3. Wash hands frequently and maintain good hand hygiene. Children should thoroughly clean their hands after going home from outside, before wearing a mask and after removing the mask, before and after meals, after contacting with animals or possibly contaminated articles, and after coughing, sneezing, and blowing their noses, to avoid contact of dirty hands with the mouth, eyes, and nose, using soap and running water. If hand washing is not applicable, disinfectant wipes or hand disinfectants containing ethanol can be used.

4. Maintaining social distance, avoid crowded places, and ensure good indoor air circulation.

5. It is necessary to have a balanced diet, maintain oral health, moderate exercise and regular routines, avoid excessive fatigue, and maintain emotional stability and mental health.

In conclusion, COVID-19 is a sudden and serious infectious disease, and the understanding of COVID-19 still needs to be deepened. Many problems such as the origin of the virus, the transmissibility and severity of after virus mutation, the response of the body's immune system after infection with the virus and vaccination, the duration of antibodies, the difference between immune response and immune protection in different populations (elderly, children, people with underlying diseases), breakthrough infection, the methods to evaluate antibody levels, and many other issues are not fully understood. Data on children are even more limited, and further in-depth studies are still needed. In addition, the research and development of vaccines against existing and future variants of SARS-CoV-2, as well as the research and development of safer, more effective and more convenient vaccines have also begun. With the approval of more COVID-19 vaccines, with accumulated clinical data of COVID-19 vaccination and data on vaccine post-marketing monitoring and evaluation, this expert consensus will be updated in time according to the needs of the epidemic prevention and control situation.

Author contributions All authors contributed equally to this paper. All authors contributed to writing of the draft and approved the final version.

Funding None.

\section{Compliance with ethical standards}

Ethical approval Not required for this editorial.

Conflict of interest All authors declared no financial or non-financial conflict of interests related to this paper.

\section{References}

1. Jiang RM, Xie ZD, Jiang Y, Lu XX, Jin RM, Zheng YJ, et al. Diagnosis, treatment and prevention of 2019 novel coronavirus infection in children: experts' consensus statement (third edition). Chin J Appl Clin Pediatr. 2021;36:721-32.

2. World health organization. WHO Coronavirus (COVID-19) dashboard. 2021. https://covid19.who.int/. Accessed 10 Sept 2021. 
3. Centers for disease control and prevention. COVID data tracker. 2021. https://covid.cdc.gov/covid-data-tracker/\#demographicsove rtime. Accessed 17 Aug 2021.

4. World health organization. WHO Coronavirus (COVID-19) dashboard. 2021.https://covid19. who.int/table. Accessed 20 Aug 2021.

5. American Academy of Pediatrics. Children and COVID-19: state-level data report. 2021. https://www.aap.org/. Accessed 20 Aug 2021.

6. Nantong Weekly. The COVID-19 vaccination in children aged 3-17 has been officially approved in China.2021. https://k.sina.cn/article_14541 05824_56abe4e0019010c8t.html?q=\$q. Accessed 20 Aug 2021.

7. Hu B, Guo H, Zhou P, Shi ZL. Characteristics of SARS-CoV-2 and COVID-19. Nat Rev Microbiol. 2021;19:141-54.

8. National Health Commission of the People's Republic of China. Novel coronavirus vaccine technical guide (first edition). Chin J Clin Infect Dis. 2021;14:89-90.

9. Sharma O, Sultan AA, Ding H, Triggle CR. A review of the progress and challenges of developing a vaccine for COVID-19. Front Immunol. 2020;11:585354.

10. Al Kaabi N, Zhang Y, Xia S, Yang YK, Qahtani MMA, Abdulrazzaq $\mathrm{N}$, et al. Effect of 2 inactivated SARS-CoV-2 vaccines on symptomatic COVID-19 infection in adults: a randomized clinical trial. JAMA. 2021;326:35-45.

11. Zhang Y, Zeng G, Pan H, Li CG, Hu YL, Chu K, et al. Safety, tolerability, and immunogenicity of an inactivated SARS-CoV-2 vaccine in healthy adults aged $18-59$ years: a randomised, doubleblind, placebo-controlled, phase 1/2 clinical trial. Lancet Infect Dis. 2021;21:181-92.

12. Jara A, Undurraga EA, González C, Paredes F, Fontecilla T, Jara $\mathrm{G}$, et al. Effectiveness of an inactivated SARS-CoV-2 vaccine in Chile. N Engl J Med. 2021;385:875-84.

13. Han B, Song Y, Li C, Yang WQ, Ma QX, Jiang ZW, et al. Safety, tolerability, and immunogenicity of an inactivated SARS-CoV-2 vaccine (CoronaVac) in healthy children and adolescents: a double-blind, randomised, controlled, phase $1 / 2$ clinical trial. Lancet Infect Dis. 2021;S1473-3099:00319-24.

14. Frenck RW Jr, Klein NP, Kitchin N, Gurtman A, Absalon J, Lockhart S, et al. Safety, immunogenicity, and efficacy of the BNT162b2 covid-19 vaccine in adolescents. N Engl J Med. 2021;385:239-50.

15. Bergwerk M, Gonen T, Lustig Y, et al. Covid-19 breakthrough infections in vaccinated health care workers. N Engl J Med. 2021. https://doi.org/10.1056/NEJMoa2109072.

16. Li XN, Huang Y, Wang W, Jing QL, Zhang CH, Qin PZ, et al. Efficacy of inactivated SARS-CoV-2 vaccines against the Delta variant infection in Guangzhou: a test-negative case-control realworld study. Emerg Microbes Infect. 2021;10:1751-9.

17. World health organization. COVID-19 vaccines technical documents (2021). https://www.who.int/groups/strategic-advisory-group-of-exper ts-on-immunization/covid-19-materials. Accessed 25 Aug 2021.

18. World health organization. Background document on mRNA vaccine BNT162b2 (Pfizer-BioNTech) against COVID-19 (2021). https://www.who.int/publications/i/item/backgrounddocument-on-mrna-vaccine-bnt162b2-(pfizer-biontech)-again st-covid-19. Accessed 25 Aug 2021.

19. World health organization. Background document on the mRNA-1273 vaccine (Moderna) against COVID-19 (2021). https://www.who.int/publications/i/item/background-docum ent-on-the-mrna-1273-vaccine-(moderna)-against-covid-19. Accessed 25 Aug 2021.

20. World health organization. Background document on the AZD1222 vaccine against COVID-19 developed by Oxford University and AstraZeneca (2021). https://www.who.int/publications/i/item/backg round-document-on-the-azd1222-vaccine-against-covid-19-devel oped-by-oxford-university-and-astrazeneca. Accessed 25 Aug, 2021

21. World health organization. Background document on the Janssen Ad26.COV2.S (COVID-19) vaccine: background document to the WHO Interim recommendations for use of Ad26.COV2.S (COVID-19) vaccine (2021). https://www.who.int/publicatio ns/i/item/WHO-2019-nCoV-vaccines-SAGE-recommendationAd26.COV2.S-background-2021.1 Accessed 25 Aug 2021.

22. World health organization. Background document on the inactivated COVID-19 vaccine BIBP developed by China National Biotec Group (CNBG) (2021). https://www.who.int/publicatio ns/i/item/WHO-2019 -nCoV-vaccines-SAGE-recommendationCOVID-19-vaccine-BIBP-background. Accessed 25 Aug 2021.

23. World health organization. Background document on the inactivated vaccine Sinovac-CoronaVac against COVID-19 (2021). https://www.who.int/publications/i/item/WHO-2019-nCoV-vacci nes-SAGE_recommendation-Sinovac-CoronaVac-background2021.1. Accessed 25 Aug 2021.

24. World Health Organization. 7 Vaccines Approved for Use by WHO. [EB/OL]. (2021-08-17). https://covid19.trackvaccines. org/agency/who/. Accessed 2 Sept 2021.

25. National Health Commission of the People's Republic of China. Transcript of the press conference of the joint prevention and control mechanism of The State Council on June 11, 2021. 2021. http://www.nhc.gov.cn/xcs/fkdt/202106/e28487f08ad745c59523 56e448a87f13.shtml. Accessed 11 Aug 2021.

26. Committee on Infectious Diseases. COVID-19 vaccines in children and adolescents. Pediatrics. 2021;148:e2021052336.

27. National Health Commission of the People's Republic of China. Transcript of the joint prevention and control mechanism of The State Council press conference on August 13, 2021. 2021. http:// www.nhc.gov.cn/xcs/fkdt/202108/6c77e46253b84b1f9cca2d5aa 4a0f76b.shtml. Accessed 13 Aug 2021.

28. National Health Commission of the People's Republic of China. Technical guidelines for vaccination of COVID-19 (version 1). Chin J Clin Infect Dis. 2021;14:89-90.

29. Shaw RH, Stuart A, Greenland M, Liu XX, Van-Tam JSN, Snape $\mathrm{MD}$, et al. Heterologous prime-boost COVID-19 vaccination: initial reactogenicity data. Lancet. 2021;397:2043-6.

30. Normark J, Vikström L, Gwon YD, Persson IL, Edin A, Björsell $\mathrm{T}$, et al. Heterologous ChAdOx1 nCoV-19 and mRNA-1273 vaccination. The N Engl J Med. 2021;9(385):1049-51.

31. Simonetti O, Rizzetto G, Molinelli E, Diotallevi F, Radi G, Cirioni O, et al. Safety and efficacy of vaccines during COVID-19 pandemic in patients treated with biological drugs in a dermatological setting. Healthcare (Basel). 2021;9:401-10.

32. Centers for disease control and prevention. Interim clinical considerations for use of COVID-19 vaccines currently authorized in the United States. [EB/OL]. [2021-09-02].https://www.cdc.gov/ vaccines/covid-19/info-by-product/clinical-considerations.html. Accessed 2 Sept 2021.

33. Polack FP, Thomas SJ, Kitchin N, Absalon J, Gurtman A, Lockhart S, et al. Safety and efficacy of the BNT162b2 mRNA Covid19 vaccine. N Engl J Med. 2020;383:2603-15.

34. Richmond P, Hatchuel L, Dong M, Ma B, Hu B, Smolenov I, et al. Safety and immunogenicity of S-Trimer (SCB-2019), a protein subunit vaccine candidate for COVID-19 in healthy adults: a phase 1, randomised, double-blind, placebo-controlled trial. Lancet. 2021;397:682-94.

35. United States centers for disease control and prevention. COVID19 vaccines for children and teens. 2021. Accessed 17 Aug 2021.

36. Chinese centers for disease control and prevention. Interpretation of adverse reaction monitoring information of Novel Coronavirus vaccine in China [EB/OL].(2021-08-17) [2021-09-02]. http:// www.chinacdc.cn/jkzt/ymyjz/ymyjjz_6758/202105/t20210528_ 230908.html. Accessed 17 Aug 2021.

Publisher's Note Springer Nature remains neutral with regard to jurisdictional claims in published maps and institutional affiliations. 


\section{Authors and Affiliations}

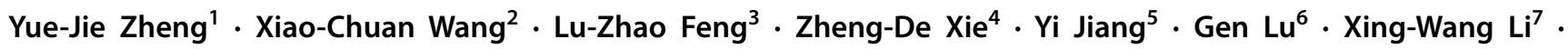
Rong-Meng Jiang ${ }^{7}$. Ji-Kui Deng ${ }^{8} \cdot$ Miao Liu ${ }^{5} \cdot$ Bao-Ping Xu ${ }^{9} \cdot$ Zhuang Wei $^{10} \cdot$ Gang Liu $^{11} \cdot$ Xiao-Xia Lu $^{12} \cdot$ Run-Ming Jin $^{13}$. Zhi-Sheng Liu ${ }^{14}$.Yun-Xiao Shang ${ }^{15}$. Sai-Nan Shu ${ }^{16} \cdot$ Yan Bai $^{13} \cdot$ Min Lu $^{17}$. Guang-Hua Liu ${ }^{18} \cdot$ Wan-Jun Luo ${ }^{19} \cdot$ Yu-Xia Cui $^{20}$. Le-Ping Ye ${ }^{21}$. Li-Kai Lin ${ }^{22}$. Dong-Chi Zhao ${ }^{23}$ - A-Dong Shen ${ }^{24}$. Jian-Bo Shao ${ }^{25}$ • Li-Juan Xiong ${ }^{26}$. Li-Wei Gao ${ }^{9}$. Tian-You Wang ${ }^{27}$. China National Clinical Research Center for Respiratory Diseases - National Center for Children's Health, Beijing, China - Group of Respirology, Chinese Pediatric Society, Chinese Medical Association - Chinese Medical Doctor Association Committee on Respirology Pediatrics . China Medicine Education Association Committee on Pediatrics - Chinese Research Hospital Association Committee on Pediatrics - Chinese Non-government Medical Institutions Association Committee on Pediatrics - China Association of Traditional Chinese Medicine, Committee on Children's Health and Medicine Research $\cdot$ Zheng-Yan Zhao ${ }^{28} \cdot$ Yong-Hong Yang ${ }^{24} \cdot$ Kun-Ling Shen ${ }^{9}$

Yong-Hong Yang

yyh628628@sina.com

$\triangle$ Kun-Ling Shen

kunlingshen1717@163.com

1 Department of Respiratory Disease, Shenzhen Children's Hospital, Shenzhen, China

2 Department of Allergy and Clinical Immunology, Children's Hospital of Fudan University, Shanghai, China

3 School of Population Medicine and Public Health, Peking Union Medical College, Beijing, China

4 Research Unit of Critical Infection in Children, Chinese Academy of Medical Sciences, 2019RU016, National Clinical Research Center for Respiratory Diseases, Beijing Pediatric Research Institute, Beijing Children's Hospital, Capital Medical University, National Center for Children's Health, Beijing, China

5 Department of Pediatrics, Renmin Hospital of Wuhan University, Wuhan, Hubei, China

6 Department of Respiratory Disease, Guangzhou Women and Children's Medical Center, Guangzhou, China

7 Diagnosis and Treatment Center of Infectious Diseases, Beijing Ditan Hospital, Capital Medical University, Beijing, China

8 Department of Infectious Diseases, Shenzhen Children's Hospital, Shenzhen, Guangdong, China

9 Department of Respiratory Disease, Beijing Children's Hospital, Capital Medical University, China National Clinical Research Center for Respiratory Diseases, National Center for Children's Health, Beijing, China

10 Children's Health Care Center, Beijing Children's Hospital, Capital Medical University, National Center for Children's Health, Beijing, China

11 Department of Infectious Diseases, Beijing Children's Hospital, Capital Medical University, National Center for Children's Health, Beijing, China

12 Department of Respiratory Disease, Wuhan Children's Hospital, Tongji Medical College, Huazhong University of Science and Technology, Wuhan, Hubei, China

13 Department of Pediatrics, Union Hospital, Tongji Medical College, Huazhong University of Science and Technology, Wuhan, China
14 Department of Neurology, Wuhan Children's Hospital, Tongji Medical College, Huazhong University of Science and Technology, Wuhan, China

15 Department of Pediatric Respiratory Disease, Shengjing Hospital of China Medical University, Shenyang, China

16 Department of Pediatrics, Tongji Hospital, Tongji Medical College, Huazhong University of Science and Technology, Wuhan, China

17 Department of Respiratory Disease, Children's Hospital of Shanghai, Shanghai, China

18 Department of Pediatrics, Fujian Branch of Shanghai Children's Medical Center, Fujian Children's Hospital, Fuzhou, China

19 Office of Healthcare-Associated Infection Management, Wuhan Children's Hospital, Tongji Medical College, Huazhong University of Science and Technology, Wuhan, China

20 Department of Pediatrics, Guizhou Provincial People's Hospital, Guiyang, China

21 Department of Pediatrics, Peking University First Hospital, Beijing, China

22 Hospital Management Institute of Wuhan University, Zhongnan Hospital of Wuhan University, Wuhan, China

23 Department of Pediatrics, Zhongnan Hospital of Wuhan University, Wuhan, China

24 Beijing Pediatric Research Institute, Beijing Children's Hospital, Capital Medical University, China National Clinical Research Center for Respiratory Diseases, National Center for Children's Health, Beijing, China

25 Department of Radiology, Wuhan Children's Hospital, Tongji Medical College, Huazhong University of Science and Technology, Wuhan, China

26 Department of Infectious Diseases, Union Hospital, Tongji Medical College, Huazhong University of Science and Technology, Wuhan, China

27 Hematology and Oncology Center, Beijing Children's Hospital, Capital Medical University, National Center for Children's Health, Beijing, China

28 Children's Hospital, Zhejiang University School of Medicine, Hangzhou, China 